\title{
ESTIMATES FOR EXPONENTIAL SUMS
}

\author{
ROBERT A. SMITH
}

\begin{abstract}
If $f$ is a polynomial over $Z$ of degree $n+1$ with $n>1$, then for each integer $q>1,\left|\Sigma_{1<x<q} \exp (2 \pi i f(x) / q)\right|\left\langle q^{1 / 2}(D, q) d_{n}(q)\right.$, provided the discriminant $D$ of the derivative of $f$ does not vanish identically, where $d_{n}(q)$ is the number of representations of $\boldsymbol{q}$ as a product of $\boldsymbol{n}$ factors.
\end{abstract}

For each positive integer $q$ and for each nonlinear polynomial $f \in \mathbf{Z}[X]$ of degree $n+1$, i.e., $n=\operatorname{deg} f-1 \geqslant 1$, we define

$$
S(f ; q)=\sum_{x \bmod q} e_{q}(f(x)),
$$

where " $x \bmod q$ " means that $x$ runs through a complete set of residues mod $q$, and $e_{q}(t)=\exp (2 \pi i t / q)$ for each $t \in \mathbf{Z}$. In 1948, A. Weil [6] proved as a consequence of his work in algebraic geometry that the exponential sum in (1) satisfies the following inequality when $q$ is a prime $p$ and $f \notin p Z[X]$ :

$$
|S(f ; p)| \leqslant(\operatorname{deg} f-1) p^{1 / 2} .
$$

For certain applications to number theory (e.g., cf. [4]), it is absolutely essential to have upper bounds for (1) with $q$ an arbitrary positive integer (and not just a prime). In 1977, Jing-Run Chen [1] proved that if the content of $f-f(0)$ is relatively prime to $q$, then (1) satisfies

$$
|S(f ; q)|<e^{7(n+1)} q^{1-1 /(n+1),}
$$

an improvement of an estimate originally due to L. K. Hua [3]. This inequality is essentially best possible (cf. [2, p. 19]). The purpose of this paper is to show that if the discriminant $D\left(f^{\prime}\right)$ of $f^{\prime}$ does not vanish identically, where $f^{\prime}$ denotes the derivative of $f$, then a substantial improvement in this estimate can be deduced from Weil's estimate in (2).

We begin by giving a new interpretation of the well-known fact that $S(f ; q)$ is multiplicative in $q$ (cf. [4, p. 2]). We observe that we may assume $f(0)=0$ without loss of generality.

THEOREM 1. Suppose $q_{1}$ and $q_{2}$ are positive integers which are relatively prime. Then there exist integers $m_{1}$ and $m_{2}$ such that

$$
m_{1} q_{1}+m_{2} q_{2}=1 \text {. }
$$

For each polynomial $f \in \mathbf{Z}[X]$ satisfying $f(0)=0$, then

$$
S\left(f ; q_{1} q_{2}\right)=S\left(m_{2} f ; q_{1}\right) S\left(m_{1} f ; q_{2}\right) .
$$

Received by the editors June 5, 1979 and, in revised form, September 10, 1979.

AMS (MOS) subject classifications (1970). Primary 10G05. 
Proor. Since the map $\left(x+q_{1} \mathrm{Z}, y+q_{2} \mathrm{Z}\right) \mapsto m_{2} q_{2} x+m_{1} q_{1} y+q_{1} q_{2} \mathrm{Z}$ defines a bijection between $\mathrm{Z} / q_{1} \mathrm{Z} \times \mathrm{Z} / q_{2} \mathrm{Z}$ and $\mathrm{Z} / q_{1} q_{2} \mathrm{Z}$, then (1) can be rewritten as

$$
S\left(f ; q_{1} q_{2}\right)=\sum_{\substack{x \bmod q_{1} \\ y \bmod q_{2}}} e_{q_{1} q_{2}}\left(f\left(m_{2} q_{2} x+m_{1} q_{1} y\right)\right)
$$

For any $x, y \in Z$, then modulo $q_{1} q_{2}$, we have

$$
\begin{aligned}
f\left(m_{2} q_{2} x+m_{1} q_{1} y\right) & \equiv f\left(m_{2} q_{2} x\right)+f\left(m_{1} q_{1} y\right) \\
& \equiv\left(m_{2} q_{2}+m_{1} q_{1}\right)\left(f\left(m_{2} q_{2} x\right)+f\left(m_{1} q_{1} y\right)\right) \\
& \equiv m_{2} q_{2} f\left(m_{2} q_{2} x\right)+m_{1} q_{1} f\left(m_{1} q_{1} y\right)
\end{aligned}
$$

(since $f(0)=0$ implies $f(q x) \equiv 0 \bmod q$ for all $x \in \mathbf{Z}$ )

$$
\begin{aligned}
& \equiv m_{2} q_{2} f\left(\left(1-m_{1} q_{1}\right) x\right)+m_{1} q_{1} f\left(\left(1-m_{2} q_{2}\right) y\right) \\
& \equiv m_{2} q_{2} f(x)+m_{1} q_{1} f(y) .
\end{aligned}
$$

This completes the proof of Theorem 1.

To establish an upper bound for $S(f ; q)$, it therefore suffices to assume that $q=p^{\alpha}$ with $\alpha>2$ in view of (2). For fixed $\alpha$, we define

$$
\delta=\left[\frac{\alpha}{2}\right] \text { and } \gamma=\alpha-\delta .
$$

Since $\alpha>2$, it follows that

$$
2 \gamma>\alpha \text { and } \gamma>\delta>1 \text {. }
$$

Furthermore, we shall assume that $f$ is a polynomial in $\mathbf{Z}[X]-p \mathbb{Z}[X]$ with $D\left(f^{\prime}\right) \neq 0$. For each pair of positive integers $r$ and $s$, then

$$
B\left(p^{r+s}\right)=p^{r} B\left(p^{s}\right) \oplus B\left(p^{r}\right)
$$

where

$$
B\left(p^{r}\right)=\left\{x \in \mathbf{Z}: 0<x<p^{r}\right\}
$$

a set of representatives of the residue classes $\bmod p^{r}$. Taking $r=\gamma$ and $s=\delta$ in (4), the Taylor expansion of $f\left(u+p^{\gamma} v\right) \bmod p^{\alpha}$ (cf. (3)) transforms (1) into

$$
S\left(f ; p^{\alpha}\right)=p^{\delta} \sum_{\substack{0<u<p^{\gamma} \\ f^{\prime}(u) \equiv 0 \bmod p^{\delta}}} e_{p^{\alpha}}(f(u)) .
$$

For each $F \in \mathbf{Z}[X]$, let

$$
N\left(F ; p^{m}\right)=\operatorname{card}\left\{x \bmod p^{m}: F(x) \equiv 0 \bmod p^{m}\right\} .
$$

By a theorem of Sándor [5], we know that if $D(F) \neq 0$, then

$$
N\left(F ; p^{m}\right)<(\operatorname{deg} F) p^{p(m, F)}
$$

where

$$
\nu(m, F)< \begin{cases}\frac{1}{2} \operatorname{ord}_{p} D(F) & \text { if } m>\operatorname{ord}_{p} D(F), \\ m-1 & \text { if } m<\operatorname{ord}_{p} D(F) .\end{cases}
$$

If $\alpha$ is even, then $\gamma=\delta>1$ whence (5) and (6) imply

$$
\left|S\left(f ; p^{\alpha}\right)\right|<n\left(D\left(f^{\prime}\right), p^{\alpha}\right) p^{\alpha / 2} .
$$


Next suppose that $\alpha$ is odd, so that $\gamma=\delta+1>2$. If ord $_{p} D\left(f^{\prime}\right)>1$, then (5) and (6) again imply that (7) holds, since $\frac{1}{2}\left(1+\operatorname{ord}_{p} D\left(f^{\prime}\right)\right)<\operatorname{ord}_{p} D\left(f^{\prime}\right)$. If $\operatorname{ord}_{p} D\left(f^{\prime}\right)$ $=0$, the decomposition in (4) (with $r=\delta$ and $s=1$ ), together with the Taylor expansion of $f\left(x+p^{\delta} y\right) \bmod p^{\alpha}$, imply that

$$
S\left(f ; p^{\alpha}\right)=p^{\delta} \sum_{\substack{0<x<p^{8} \\ f^{\prime}(x) \equiv 0 \bmod p^{\delta}}} e_{p^{\alpha}}(f(x)) \sum_{0<y<p} e_{p}\left(\frac{1}{2} f^{\prime \prime}(x) y^{2}+p^{-\delta} f^{\prime}(x) y\right) .
$$

If $p>2$, the absolute value of the Gaussian sum in (8) is $p^{1 / 2}$ since ord $p\left(f^{\prime}\right)=0$, whence $\left|S\left(f ; p^{\alpha}\right)\right|<n p^{\alpha / 2}$, and similarly for $p=2$. Therefore, we have proved that for all $\alpha>2$ and for all $f \in \mathbf{Z}[X]-p \mathbb{Z}[X]$ for which $D\left(f^{\prime}\right) \neq 0$, the inequality in (7) holds. We can now prove

THEOREM 2. Suppose $f$ is a nonlinear polynomial in $\mathbf{Z}[X]$ such that $D\left(f^{\prime}\right) \neq 0$. Then for any integer $q>1$,

$$
|S(f ; q)|<q^{1 / 2}\left(D\left(f^{\prime}\right), q\right) d_{n}(q),
$$

where $n=\operatorname{deg} f-1>1$ and $d_{n}(q)$ denotes the number of representation of $q$ as $a$ product of $n$ factors.

Proop. First, we shall assume that $q=p^{\alpha}$, where $p$ is a prime. Clearly, there exists a unique integer $t>0$ and a unique polynomial $g \in \mathbb{Z}[X]-p \mathbb{Z}[X]$ such that

$$
f(X)=p^{t} g(X) \text {. }
$$

If $t>\alpha$, then (1) implies $S\left(f ; p^{\alpha}\right)=p^{\alpha}$, which certainly satisfies the inequality (7) since

$$
D(v F)=v^{2 \operatorname{deg} F-1} D(F)
$$

for any $F \in \mathbf{Z}[X]$ and any $v \in \mathbf{Z}$. If $t<\alpha$, then (1) implies that

$$
S\left(f ; p^{\alpha}\right)=p^{t} S\left(g ; p^{\alpha-t}\right) .
$$

If $t=\alpha-1$, then (11), together with (2), imply that $\left|S\left(f ; p^{\alpha}\right)\right|<n p^{t+1 / 2}$, i.e., the inequality (7) is again satisfied in view of (10). Thus, we may assume $\alpha-t>2$. By what has already been proved in (7), we have

$$
\left|S\left(g ; p^{\alpha-t}\right)\right|<n\left(D\left(g^{\prime}\right), p^{\alpha-t}\right) p^{(\alpha-t) / 2},
$$

whence $S\left(f ; p^{\alpha}\right)$ again satisfies the inequality (7) in view of (9), (10) and (11). Hence, we have shown that (7) holds under the assumptions of Theorem 2.

Now let $q>1$ be arbitrary. Without loss of generality, we may assume that $f(0)=0$. By Theorem 1 ,

$$
S(f ; q)=\prod_{p^{\alpha} \| q} S\left(m\left(p^{\alpha}\right) f ; p^{\alpha}\right),
$$

where $m\left(p^{\alpha}\right)$ is a suitable integer satisfying

$$
\left(m\left(p^{\alpha}\right), p\right)=1
$$

for each prime $p$ dividing $q$. Thus, (12) implies

$$
|S(f ; q)|<\prod_{p^{\alpha} \| q} n\left(D\left(m\left(p^{\alpha}\right) f^{\prime}\right), p^{\alpha}\right) p^{\alpha / 2}<d_{n}(q)\left(D\left(f^{\prime}\right), q\right) q^{1 / 2}
$$


in view of (10) and (13), together with the fact that

$$
\prod_{p \mid q} n=\prod_{p \mid q} d_{n}(p)<d_{n}(q)
$$

This completes the proof of Theorem 2.

REMARK. If $\delta>\operatorname{ord}_{p} D\left(f^{\prime}\right)>1$, we observe that the inequality (7) can be replaced by the stronger inequality (cf. (6))

$$
\left|S\left(f ; p^{\alpha}\right)\right|<n\left(D\left(f^{\prime}\right), p^{\alpha}\right)^{1 / 2} p^{\alpha / 2} .
$$

It is therefore reasonable to ask if (14) holds for $\delta<\operatorname{ord}_{p} D\left(f^{\prime}\right)$ whenever $\operatorname{ord}_{p} D\left(f^{\prime}\right)>1$. It appears that such an improvement would require a very detailed analysis of the auxiliary exponential sum in (5) for those primes $p$ dividing the discriminant of $f^{\prime}$ (there are only a finite number of such primes!). Thus, if (14) holds for all primes $p$ dividing $D\left(f^{\prime}\right)$, then the inequality in Theorem 2 can be strengthened to

$$
|S(f ; q)| \leqslant q^{1 / 2}\left(D\left(f^{\prime}\right), q\right)^{1 / 2} d_{n}(q)
$$

\section{REFERENCES}

1. Jing-Run Chen, On Professor Hua's estimate of exponential sums, Sci. Sinica 20 (1977), 711-719.

2. G. H. Hardy and J. E. Littlewood, Some problems of "Partitio numeronum": II. Proof that every large number is the sum of at mast 21 biquadrates, Math. Z. 9 (1921), 14-27.

3. L. K. Hua, On an exponential sum, J. Chinese Math. Soc. 2 (1940), 301-312.

4. , Additive theory of prime numbers, Transl. Math. Mono., vol. 13, Amer. Math. Soc., Providence, R. I., 1965.

5. G. Sándor, Über die Anzahl der Lösungen einer Kongruenz, Acta Math. 87 (1952), 13-17.

6. A. Weil, On some exponential sums, Proc. Nat. Acad. Sci. U.S.A. 34 (1948), 204-207.

Department of Mathematics, Universtry of Toronto, Toronto M5S 1A1, Canada 